\title{
"A Study of Tru-Cut Biopsy and Fnac in a Clinically Palpable Breast Lump"
}

\author{
Ganesh Gojanur ${ }^{1}$, Lancelot Lobo ${ }^{2}$,Nakul Mahesh Babu ${ }^{3}$,Adithya $\mathrm{G}^{4}$ \\ 2 (Associate Professor, Department Of Surgery K.S.Hegde Medical Academy, Nitte University, Mangalore) \\ ${ }^{1,3}$ (Residents, Department Of Surgery K.S.Hegde Medical Academy, Nitte University, Mangalore \\ ${ }^{4}$ (Senior Resident, Department Of Surgery K.S.Hegde Medical Academy, Nitte University, Mangalore)
}

\begin{abstract}
Background: Breast is host to many diseases which range from benign and malignant neoplasm's, inflammatory conditions to infections, most of which present as lumps in the breast. Breast lumps are one of the commonest complains encountered in surgical OPD's which makes it important to differentiate between benign and malignant conditions before treating it. The successful diagnosis of the disease is based on a thorough understanding of normal anatomy, physiologic and pathologic features of the breast.

Success in surgical management of patient can be conveniently divided into three phases Pre, intra and post operative. Vitally important is the preoperative decision making and planning. An expertly performed operation for a wrong reason is still a bad operation. The foundation of this success is thorough knowledge of surgical anatomy and pathology. Various diagnostic methods have been developed to evaluate the breast lumps with the goal of identifying a sensitive, specific, efficient and economical approach to diagnose breast cancer. Physical examination, mammography, Tru-cut biopsy (Core needle biopsy), ultrasonography, thermography, FNAC open excision biopsy are all used to greater or lesser extent in diagnostic workup of a palpable breast mass. Various combinations of these approaches have been studied and have been found to increase the specificity and sensitivity over that of any one test alone.

Fine Needle Aspiration Cytology has been proved to be of great value in the diagnosis of breast lumps, apart from being cost effective, it is also simple and quick while providing the cytological diagnosis. FNAC is often used as a first priority investigation in patients with breast lump.

zobtaining histopathological diagnosis. It is relatively easy and can be performed on an outpatient basis. It also avoids unnecessary excisional biopsy.

This study is aimed to find out the sensitivity and specificity of FNAC and Tru-cut biopsy with respect to clinically palpable breast lumps by comparing it with the histopathological (gross specimen) reports.
\end{abstract}

Aims and Objectives of the study.

1. To find the sensitivity and specificity of FNAC and Tru-cut biopsy

2. To study the efficacy of FNAC and Tru-cut biopsy with that of

Histopathological (gross specimen) study in diagnosing a breast lump.

3. To find limitations of FNAC and Tru-cut biopsy.

Material and methods: A total number of 30 FNAC and 30 Tru-cut biopsies of breast collected from July 2014 to June 2016 at KSHEMA Hospital, Mangalore.

Results: We had accuracy rate of $86.4 \%$ for FNAC, $96.3 \%$ for True-Cut biopsy and combined accuracy of $100 \%$. The overall sensitivity of FNAC is 76.8\% and that of True cut biopsy is 90\% in our study. The overall specificity of FNAC is 100\% and that of True cut biopsy is $100 \%$ in our study. The overall sensitivity of FNAC and true cut biopsy combined is $100 \%$ in our study. The overall specificity of FNAC and True cut biopsy combined is $100 \%$ in our study

Conclusion: Both FNAC and True cut biopsy are important tool in management of patients with breast lump. When performed by expert hands, the diagnostic accuracy of both is very high but not 100\%.when combined together accuracy is $100 \%$.

Thus we have no hesitation that FNAC and True cut biopsy is a very preliminary diagnostic test in clinically palpable breast lumps, the results show 100\% co relation with postoperative histopathology report when 
combined together so it can be advised that the patients who have undergone both the above preliminary diagnostic procedures can be directly managed with definitive therapy.

Keywords: Fnac,Tru Cut Biopsy,Breast Lump,Histopath Report.

\section{Introduction}

Breast diseases includes a variety of lesions including inflammatory, neoplastic and hormonal response disorders. The majority of the lesions that occur in the breast are benign. However much concern is given to the malignant lesions of the breast, as breast malignancy is one of the commonest malignancy in women throughout the world. ${ }^{1}$

Breast lungs constitutes significant number of cases seen in surgical cut patient departments and frequently the need arises to distinguish between benign and malignant lumps prior to definitive treatment. ${ }^{2}$

In recent past FNAC and Tru-cut biopsy has replaced incisional or excisional biopsy in diagnosing a breast lumph, because of its distinct advantages like out patient procedure, cost effectiveness, no need general anaesthesia less pain compared to incisional and excisional biopsy. ${ }^{2}$

Sometimes it is difficult to determine whether a suspicious lump in the breast is benign or malignant, simply with clinical examination and FNAC. Hence, combination of various different investigations arise frequently.

FNAC and Tru-cut biopsy are complementary procedures ${ }^{3,4}$ Pinder and Associated (19960) and Masood $(1996)^{3}$ have stated there is insufficient evidence to decide if one method is better than another. These authors recommended the use of the appropriate combination of FNAC and/or Tru-cut biopsy as the best approach for he diagnosis of breast lesions at difficult settings.

So, the purpose of this study it to see and evaluate our experience with FNAC and Tru-cut biopsy in a series of patients and compare the results with post operative final diagnosis.

\section{Materials And Methods}

Method of study:

Prospective study of patients admitted in KSHEMA hospital with lump in the breast.

Setting:

Department of General Surgery, KSHEMA Hospital.

Methodology:

Detailed history was taken from the patients. Thorough General Physical Examination, local examination and systemic examination was carried out in each patient and entered in Performa. All the patients underwent FNAC and Tru-cut Biopsy following informed consent and findings were documented. All the patients were subjected to Surgery and post operativehisto pathological diagnosis was documented and compared to preoperative findings of FNAC and Tru-cut biopsy.

Duration of study:

2 year (2014-2016)

Sample size:

30 cases

Inclusion criteria:

All female patients above the age of 20 years with a clinically palpable breast swelling.

Exclusion criteria:

1. Patients below 20 years of age.

2. Patients with breast lump who are not taken up for surgery.

3. Patients non-compliant for FNAC or Tru-cut biopsy.

4. Male patients.

5. Patients who are undergoing Neoadjuvant therapy .

III. Method Of Dat A Collection

The results of this study were calculated by using the methodology of Galen and Gambino for substantiating the correlation

$\mathrm{TP}$ - True positive

TN- True negative

FP - False positive

FN - False negative

The stastistical data was analysed using

Sensitivity $=$ TP $/ \mathrm{TP}+$ FN $\times 100$

Specificity $=\mathrm{TN} / \mathrm{TN}+\mathrm{FP} \times 100$

Positive predictive value $=\mathrm{TP} / \mathrm{TP}+\mathrm{FP} \times 100$ 
Negative predictive value $=\mathrm{TN} / \mathrm{TN}+\mathrm{FN} \times 100$

Efficacy $=\mathrm{TP}+\mathrm{TN} / \mathrm{TP}+\mathrm{FN}+\mathrm{FP}+\mathrm{TN} \times 100$

\section{Results}

Age: Out of 30 women studied ,age incidence was ranged between 23 to 76 years (mean age 43.76 years) The age incidence for the benign breast lesions ranged from 23 to 51 years (mean age 32.37years). The incidence for malignant breast lesion ranged from 34 to 76 years (mean age 50.74Years)

\begin{tabular}{|l|l|l|l|}
\hline \multicolumn{2}{|l|}{ Age } & Frequency & Percent \\
\hline \multirow{4}{*}{} & $21-30$ & 5 & 16.7 \\
\cline { 2 - 4 } & $31-40$ & 9 & 30.0 \\
\cline { 2 - 4 } & $41-50$ & 10 & 33.3 \\
\cline { 2 - 4 } & $51-60$ & 4 & 13.3 \\
\cline { 2 - 4 } & $61-70$ & 2 & 6.7 \\
\cline { 2 - 4 } & Total & 30 & 100.0 \\
\hline
\end{tabular}

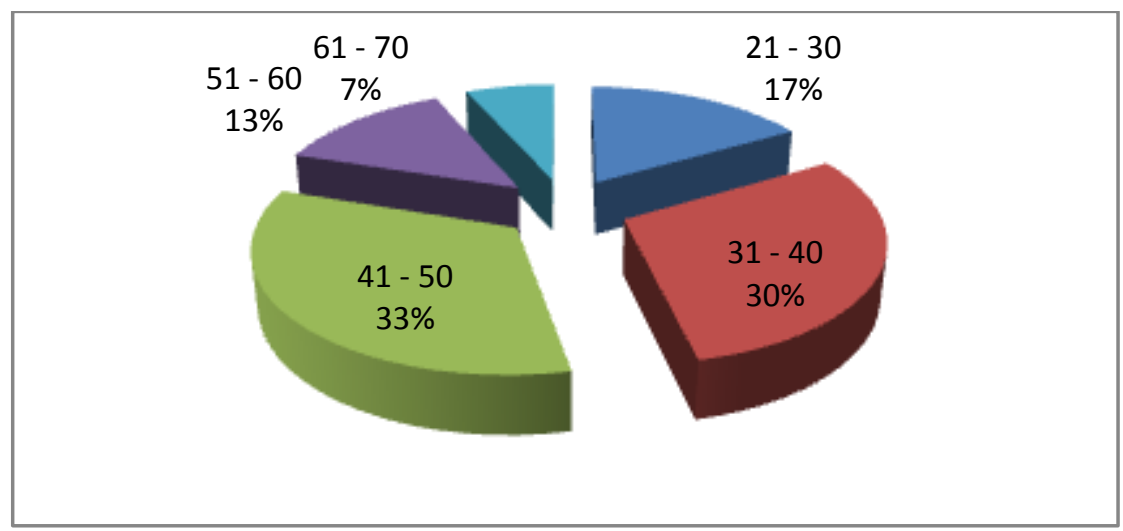

$3^{\text {rd }}$ decade of life is the most common age group for benign breast lesions

$5^{\text {th }}$ decade of life was found to be most common age group for malignant breast lesions.

Out of 30 women studied, 16 had a lump in right breast $53.3 \%, 14$ had lump in left breast $46.7 \%$.

\section{Size Of Lump}

The size of the breast lump ranged from $4 \mathrm{X} 3 \mathrm{~cm}$ to $20 \mathrm{X} 10 \mathrm{cms}$. The Benign lesions ranged from $4 \mathrm{X} 3 \mathrm{cms}$ to $20 \mathrm{X} 10 \mathrm{cms}$. Malignant lesions were ranged between $5 \mathrm{X} 4 \mathrm{~cm}$ to $18 \mathrm{X} 12 \mathrm{cms}$.

\begin{tabular}{|l|l|l|l|}
\hline \multicolumn{2}{|l|}{ Size } & Frequency & Percent \\
\hline \multirow{4}{*}{} & 5 and below & 14 & 46.7 \\
\cline { 2 - 4 } & $>5 \mathrm{~cm}$ & 16 & 53.3 \\
\cline { 2 - 4 } & Total & 30 & 100.0 \\
\hline
\end{tabular}

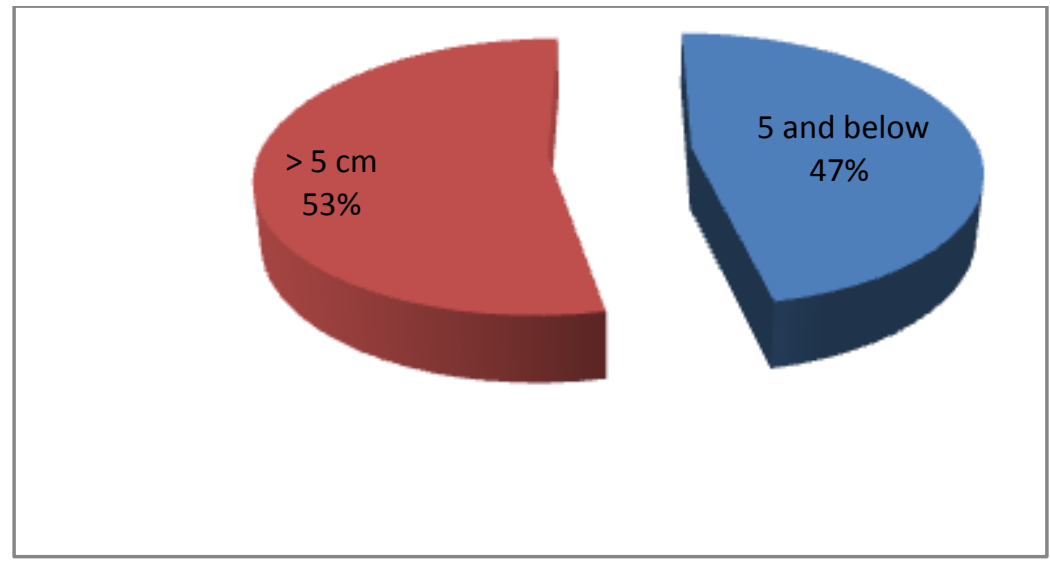




\begin{tabular}{|l|l|l|l|}
\cline { 2 - 4 } \multicolumn{1}{c|}{} & \multicolumn{3}{|l|}{ Confidence Interval } \\
\hline & Estimate & Lower & Upper \\
\hline Sensitivity & 55.00 & 33.20 & 76.80 \\
\hline Specificity & 100.00 & 100.00 & 100.00 \\
\hline PPV & 100.00 & 100.00 & 100.00 \\
\hline NPV & 52.63 & 30.18 & 75.08 \\
\hline Overall accuracy** & 70.00 & 53.60 & 86.40 \\
\hline & Value & $\mathrm{p}=$ & \\
\hline Kappa & 0.449 & 0.003 & HS \\
\hline
\end{tabular}

Duration

Duration of symptoms varied from 5 to 36 months. The mean duration of symptoms for benign lesions was 13 months , for malignant lesions was 9.7 months and overall was 11.04 months.

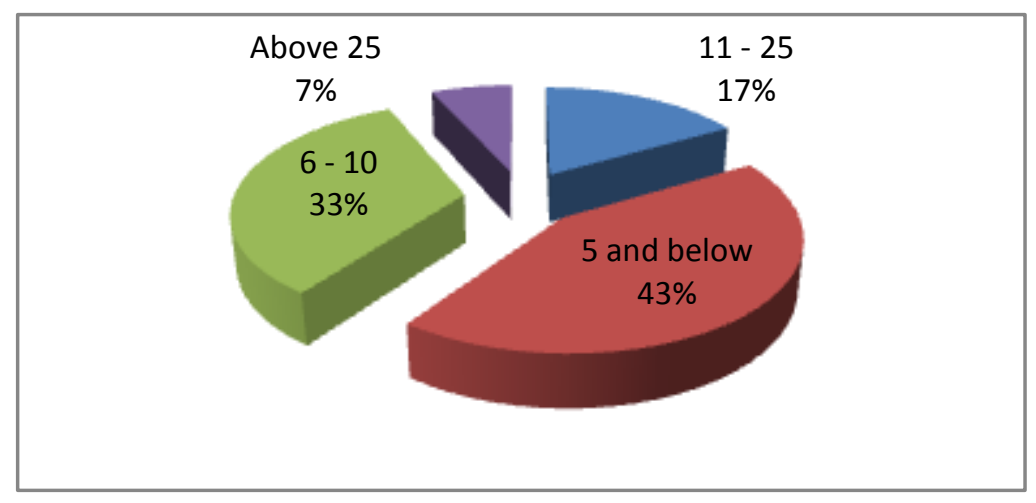

Fnac

\begin{tabular}{|l|l|l|l|}
\hline \multicolumn{2}{|l|}{ FNAC } & Frequency & Percent \\
\hline \multirow{2}{*}{ Malignant } & 17 & 64.7 \\
\cline { 2 - 4 } & Benign & 13 & 35.3 \\
\cline { 2 - 4 } & Total & 30 & 100.0 \\
\hline
\end{tabular}

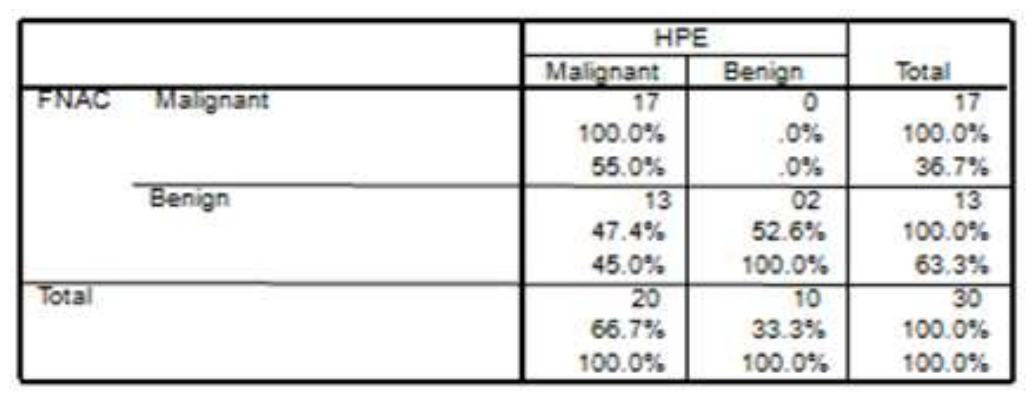

Out of 30 FNAC we got 17 positive and 13 malignant. with p value of 0.003 which is significant.

\section{Tru-Cut}

\begin{tabular}{|l|l|l|l|}
\hline \multicolumn{2}{|l|}{ TRU-CUT } & Frequency & Percent \\
\hline \multirow{3}{*}{} & Malignant & 20 & 60.0 \\
\cline { 2 - 4 } & Benign & 10 & 40.0 \\
\cline { 2 - 4 } & Total & 30 & 100.0 \\
\hline
\end{tabular}




\begin{tabular}{|c|c|c|c|}
\hline & \multicolumn{2}{|c|}{ HPE } & \multirow[b]{2}{*}{ Total } \\
\hline & Malignant & Benign & \\
\hline \multirow[t]{3}{*}{ TRU-CUT Malignant } & 20 & 0 & 20 \\
\hline & $100.0 \%$ & $.0 \%$ & $100.0 \%$ \\
\hline & $96.0 \%$ & $.0 \%$ & $60.0 \%$ \\
\hline \multirow[t]{3}{*}{ Benign } & 0 & 10 & 10 \\
\hline & $0 \%$ & $83.3 \%$ & $100.0 \%$ \\
\hline & $0 \%$ & $100.0 \%$ & $40.0 \%$ \\
\hline \multirow[t]{3}{*}{ Total } & 20 & 10 & 30 \\
\hline & $66.7 \%$ & $33.3 \%$ & $100.0 \%$ \\
\hline & $100.0 \%$ & $100.0 \%$ & $100.0 \%$ \\
\hline
\end{tabular}

\begin{tabular}{|l|l|l|l|}
\cline { 2 - 4 } \multicolumn{1}{c|}{} & & \multicolumn{2}{l|}{ Confidence Interval } \\
\hline & Estimate & Lower & Upper \\
\hline Sensitivity & 90.00 & 76.85 & 100.00 \\
\hline Specificity & 100.00 & 100.00 & 100.00 \\
\hline PPV & 100.00 & 100.00 & 100.00 \\
\hline NPV & 83.33 & 62.25 & 100.00 \\
\hline Overall accuracy** & 96.33 & 84.41 & 100.00 \\
\hline & Value & $\mathrm{p}=$ & \\
\hline Kappa & 0.857 & 0.000 & HS \\
\hline
\end{tabular}

\begin{tabular}{|l|l|l|}
\hline HPR & Frequency & Percent \\
\hline Malignant & 20 & 66.7 \\
\hline Benign & 10 & 33.3 \\
\hline Total & 30 & 100.0 \\
\hline
\end{tabular}

\begin{tabular}{|l|l|l|}
\hline Frequency & Percent & \multicolumn{2}{l|}{} \\
\hline Fibroadenoma & 6 & 16.7 \\
\hline Granulomatous & 1 & 3.3 \\
\hline $\begin{array}{l}\text { Infiltrating } \\
\text { Ductal Ca }\end{array}$ & 14 & 47.0 \\
\hline $\begin{array}{l}\text { Infiltraing Lobular } \\
\text { Ca }\end{array}$ & 6 & 20.0 \\
\hline Phyloides & 3 & 13.0 \\
\hline Total & 30 & 100.0 \\
\hline
\end{tabular}

\section{Discussion}

In present study which was conducted on 30 female patients who presented with clinically palpable breast lump all the patients underwent FNAC and Tru-cut biopsy of the lump followed by surgery in the form of definitive surgical procedures. On basis of FNAC and Tru-cut biopsy a preparative diagnosis was attained which was then finally compared with the post surgery histopathology, to see the accuracy of FNAC and Trucut biopsy and accuracy of both when combined together.

Our study of 30 patients, age incidence was ranged from 23 to 76 years (mean age 43.76 years) and most common age group having breast lump was 31 to 50 years. The incidence for benign breast lesions ranged from 23 to 51 years (mean age 32.37 years). The incidence of malignant lesions ranged from 34 to 76 years (mean age 50.74 years). The most common age group for benign lesions is 21 to 30 years and that of malignant is 41 to 50 years. The study done by A. Khenka et al ${ }^{37}$, the age range was 14 to 61 years with the overall mean age was 37.5 years, peak incidence for the benign lesion in $2^{\text {nd }}$ and $3^{\text {rd }}$ decade of life respectively 
and malignant lesions above the age of 40 years and peak incidence in between $40-44$ years. Similar studies by Homesh et al, ${ }^{38}$ Tiwari,39 Alam et $\mathrm{al}^{40}$ and Gimire et $\mathrm{al}^{41}$ showed similar age patterns.

In our study out of 30 patients, 13 patients had lump in right breast, 17 had lump in left breast. Benign lesions were found more common in the left breast, malignant lesions were found more common in the right breast in present study.

A Khemka et al $^{17}$ in their study showed left breast involvement in 28 patients while the other 22,right breast involved. No surgical importance can be derived from this observation since patient selection was in no way dictated by involvement of side of breast.

Quadrant of the breast involved in our study, shows that maximum 18(36\%) cases out of 30 had lump in the upper outer quadrant of the breast, maximum 8 out of 10 benign cases had lump in lower outer quadrant and maximum of 15 out of 31 malignant cases had lump in upper outer quadrant. O.N. Alema et $\mathrm{al}^{42}$, out of total 82 cases 40 cases had lump in upper outer quadrant. O.N. Alema et al ${ }^{42}$, out of total 85 cases 49 cases had lump in upper outer quadrant and out of them 6 cases were malignant in this study malignancy was not found in other quadrants of breast. Hussain43, in his series, had 29 patients with a lump in the upper outer quadrant and 9 had a lump in the upper inner quadrant while 4 patients had a palpable lump in lower quadrants.

Duration of symptoms in our study shows maximum 21((42\%) out of all cases presented between 7 to 12 months of duration (mean 11.04 months). The maximum of 13 benign lumps out of 19 presented between 7 to 18 months duration (mean 13 months) and the maximum of 13 malignant lumps out of 13 presented between 7 to 12 months duration (mean 9.7 months). The study by Hussain shows similar kind of findings in his study, duration in his study ranged between 1 month to 2 years with majority of patients having a history of between 6 months to 1 year.

The commonest benign pathology found in our patients was fibroadenoma almost $58 \%$ of all benign diagnosis. And the commonest malignant diagnosis was infiltrative ductal carcinoma $412 \%$ of all malignant conditions. Tiwari at al in their study of 91 patients also reported fibroadenoma as the predominance benign diagnosis. A Khenika et $\mathrm{al}^{37}$ in their study, the commonest pathology found was fibroadenoma in 29 patients. Sumaira Zareef et $\mathrm{al}^{44}$, and Ashwin ${ }^{45}$ also found fibroadenoma as commonest benign lesion.

\section{Fnac \& Tru-Cut Biopsy}

In our study True positive for FNAC was 45(90\%) True negative was 50 (100\%) and false positive was zero and false negative was 05 (10\%), which lead to the interpretation of sensitivity of $90 \%$ for FNAC and specificity of $100 \%$ for FNAC. For FNAC in our study positive and negative predictive value was found to be $100 \%$ and $90.9 \%$ respectively, with an accuracy rate of $95 \%$

Our study True positive for True-cut biopsy was 47(94\%) True negative was 49(98\%) and False positive was $01(02 \%$ ) and false negative was $03(06 \%)$, which lead to the interpretation of sensitivity of $94 \%$ for Trucut biopsy and specificity of $98 \%$ for Tru cut biopsy. For Tru-cut biopsy in our study positive and negative predictive value was found to be $97.91 \%$ and $94.23 \%$ respectively, with an accuracy rate of $96 \%$.

In a series of 100 patients, Dennison46, reported the sensitivity of FNAC as $90.4 \%$ whereas an absolute value of $90.9 \%$ was obtained by the series by Hussain et al. Westend et $\mathrm{al}^{47}$, depicted, a value of $92 \%$ for FNAC and Ashwin mentioned sensitivity as $93.10 \%$. In his study $\mathrm{Wu}$ et al ${ }^{49}$ compared postoperative pathology results and showed a sensitivity of $75 \%$ while Tru-cut biopsy showed a sensitivity value of $92 \%$. Randa et al ${ }^{49}$ mentioned in their study that the overall sensitivity, specificity and accuracy of FNAC were 56., 809 and 63.8 respectively while those of Tru-cut biopsy (histopathology) were 93.5, 83.5 and $89.7 \%$ respectively, almost similar results were found in the study done by saleh FM et al. ${ }^{50}$.

Conclusion

Both FNAC and True cut biopsy are important tool in management of patients with breast lump. When performed by expert hands, the diagnostic accuracy of both is very high but not $100 \%$.when combined together accuracy is $100 \%$.

Infiltrating ductal carcinoma was predominant malignant breast tumour in our study $47 \%$, majority were found in age group of $41-50$ years. Fibroadenoma was the commonest benign breast tumour in our study $16.7 \%$,majority with age group $21-30$ Years.

Thus we have no hesitation that FNAC and True cut biopsy is a very preliminary diagnostic test in clinically palpable breast lumps, the results show $100 \%$ co relation with postoperative histopathology report when combined together so it can be advised that the patients who have undergone both the above preliminary diagnostic procedures can be directly managed with definitive therapy.

\section{References}

[1]. Brancato B. Accuracy of needle biopsy of breast lesions visible on ultrasound:audit of fine needle versus core needle biopsy in 3233 consecutive samplings with ascertained outcomes. Breast 2012 Aug:21(4):449-54.

[2]. S M Willems, C H M van Deurzen, P J van Diest. Diagnosis of breast lesions: fine-needle aspiration cytology or core needle biopsy? A review. Group.bmj.com 2011 Oct29. 
[3]. Poole G H. Diagnosis of breast cancer with core-biopsy and fine needle aspiration cytology. Australian and New Zealand Journal of Surgery 1996 Sep Vol.66 Issue 9:592-594.

[4]. Zardawi I M. The role and histological classification of needle core biopsy in conjunction with fine needle aspiration cytology in the preoperative assessment of breast lesions. Australian Journal 2001Feb.

[5]. Westenend P J, Sever A R, Beekman H J C, Liem S J. A comparison of aspiration cytology and core needle biopsy in the evaluation of breast lesions. Cancer Cytopathology 2001 Feb Vol.93 Issue 2:146-150.

[6]. Farzana Memom, Sajida Qureshi. Sensitivity of fine needle aspiration vs. core biopsy in the diagnosis of palpable and clinically suspicious breast lesions. Pakistan Journal of surgery 2009 Vol.25 Issue 3:214-217.

[7]. Bukhari M H, Arshad M, Jamal Shahid. Use of fine-needle aspiration in the evaluation of breast lumps. Pathology research international. 2011 Article ID 689521, 10 pages.

[8]. Richards Sainsbury. The Breast. Bailey and Love's short practice of surgery. 2008 Edition 25:827-848.

[9]. Kelly K Hunt, Lisa A Newman, Edward M Copeland III, Kirby I Bland. The Breast. Schwartz's prrinciples. 